\title{
Romboencefalitis por Listeria monocytogenes. Patología emergente en relación al brote epidémico. Presentación de tres casos clínicos
}

\author{
Gonzalo Miranda $\mathrm{G}^{1}$, Patricia O rellana $\mathrm{P}^{1}$, Holvis Dellien $\mathrm{Z}^{1,2}$, \\ Margarita Switt R ${ }^{1 a}$. \\ Listeria monocytogenes \\ rhomboencephalitis. \\ Report of three cases
}

An unusual number of cases of rhomboencephalitis have occurred in Chile as a consequence of the increased frequency of infections caused by Listeria monocytogenes. We report three females aged 36, 40 and 55 years, with the disease. All presented with a prodrome characterized by headache, nausea, vomiting and fever, followed by ataxia and unilateral palsies of the third, seventh and twelfth cranial nerves. One patient presented also a hemi-hypoesthesia. Magnetic resonance showed lesions in the posterior aspect of the brain stem, specifically in relation to the floor of the fourth ventricle. Blood cultures were positive for Listeria monocytogenes (Rev Méd Chile 2009; 137: 1602-6).

(Key words: Bacteremia; Encephalitis; Listeria monocytogenes)

\author{
Recibido el 6 de marzo, 2009. Aceptado el 28 de septiembre, 2009. \\ ${ }^{1}$ Servicio de Imagenología. Hospital Clínico de la Universidad de Chile. ${ }^{2}$ Servicio de \\ Imagenología. Clínica Indisa \\ aPrograma de formación de especialista en Imagenología, Universidad de Chile
}

\begin{abstract}
L as infecciones por Listeria monocytogenes se conocen desde hace mucho tiempo. Se han comunicado diversos brotes de listeriosis, especialmente después de la década 1960-69, posiblemente en relación a la introducción y masificación de la refrigeración de los alimentos ${ }^{1}$. Esta bacteria, un coco bacilo Gram positivo, es encontrada en los más variados ambientes, no siendo raro su
\end{abstract}

Correspondencia a: Gonzalo Miranda González. Teléfonos: (02) 4759426 (07) 4953643. Fono Fax: 9789191.

E mail: gomiranda2000@yahoo.com aislamiento en alimentos refrigerados, especialmente de lácteos y carnes, en porcentajes que varían del $15 \%$ a $70 \%{ }^{2}$. Se considera en países desarrollados una de las principales infecciones adquiridas por los alimentos. Si bien lo anterior, los casos de listeriosis no son frecuentes, considerándose una patología rara, con una incidencia anual estimada de 0,3/100.000 habitantes ${ }^{3}$. La infección del huésped depende de muchos factores, no completamente establecidos, pero que tendrían relación con la cantidad de microorganismos ingeridos, el pH gástrico y la susceptibilidad del huésped. Esto último es muy importante 
considerándose pacientes más susceptibles a las mujeres embarazadas, niños y personas con inmunodepresión de diversas causas 3 .

La listeria puede comprometer diversos órganos teniendo particular predilección por el sistema nervioso central. Es así que la presentación más frecuente sea una meningitis. Pero también han sido descritos casos de meningoencefalitis, romboencefalitis, abscesos cerebrales, endocarditis, osteomielitis, artritis, pleuritis y peritonitis ${ }^{4,5}$. En pacientes inmunocompetentes se puede manifestar como una gastroenteritis aguda febril ${ }^{4,6}$.

Una particular forma de infección es la romboencefalitis que traduce compromiso inflamatorio del tronco cerebral y cerebelo. La romboencefalitis tiene diversas causas ${ }^{7}$, especialmente en niños, se ha descrito secundaria a cuadros virales (adenovirus, virus herpes simple) y en general tienen un buen pronóstico. En adultos se asocia principalmente a herpes simple y Listeria. La romboencefalitis por Listeria monocytogenes en adultos se asocia a una alta morbi-mortalidad ${ }^{8,9}$ afectando tanto a pacientes inmunocompetentes como inmunosuprimidos ${ }^{7}$. Algunas series comunican una mortalidad de hasta $61 \%$, pese al tratamiento antibiótico $^{8}$. El tratamiento de elección es ampicilina asociado o no a un aminoglucósido por una duración de dos a tres semanas ${ }^{8}$. En todos los casos publicados se han podido establecer características clínicas e imagenológicas que con un cierto grado de sospecha clínica permiten establecer un diagnóstico precoz.

\section{CASOS CLÍNICOS}

De los tres pacientes presentados ninguno presentaba una patología predisponente, siendo los tres casos mujeres, cuyas edades correspondían a 36, 40 y 55 años, respectivamente.

En los tres casos revisados se pudo establecer un cuadro clínico prodrómico caracterizado por cefalea, náuseas, vómitos, compromiso del estado general y sensación febril. Un caso se presentó inicialmente con un leve compromiso de conciencia y posteriormente también refirió sensación febril. Posterior a los síntomas descritos, que tuvieron una duración de aproximadamente una semana, se agregaron síntomas y signos de compromiso del tronco cerebral, caracterizados por compromiso unilateral de los pares craneanos (que indistintamente comprometió a los pares III, VII y XII), ataxia y en un caso compromiso de vías largas con hemihipoestesias. Ante las alteraciones previamente descritas se realizaron estudios de laboratorio e imágenes.

Estudio de líquido céfalo raquídeo (LCR). De los diversos exámenes realizados cabe consignar los estudios LCR, que en uno de los casos fue normal, y en los otros dos casos mostró alteraciones inespecíficas caracterizados por una elevación de las proteínas ( $77 \mathrm{mg} / \mathrm{dl}$ en el primer caso, $88 \mathrm{mg} / \mathrm{dl}$ en el segundo caso) y de los leucocitos ( $75 / \mathrm{mm}^{3}$, con $80 \%$ PMN en un caso, recuento normal en los otros dos casos). Todos los cultivos de LCR fueron negativos para Listeria monocytogenes.

Estudio imagenológico por resonancia magnética (RM). Los estudios imagenológicos por RM mostraron lesiones que característicamente comprometían el aspecto posterior del tronco cerebral, específicamente en relación al piso del cuarto ventrículo con un diámetro mayor en sentido longitudinal (Figuras 1 y 2). En cuanto a su señal estas lesiones fueron hiperintensas en secuencias $\mathrm{T} 2 \mathrm{y}$ discretamente hipointensas en secuencias T1. Tras la administración de medio de contraste se observó un realce homogéneo de las lesiones, que en un caso evolucionó a realce en anillo, compatible con la formación de un absceso. En difusión las imágenes mostraron una discreta hiperintensidad de señal, que en el caso del absceso mencionado previamente mostró una clara restricción a la difusión con marcada hiperseñal (Figura 2). En secuencias T2 de gradiente (no mostrada) no se detectaron artefactos de susceptibilidad magnética sugerente de hemorragia o calcificación.

Estudio bacteriológico. Ante la sospecha clínica de romboencefalitis secundaria a Listeria monocytogenes sumado a los hallazgos imagenológicos, se completó el estudio con hemocultivos, resultando los tres casos positivos, corroborándose de esta forma la sospecha clínica. Es importante la sospecha clínica para realizar el estudio bacteriológico dado que los cultivos de LCR para Listeria monocytogenes tienen en general una baja sensibilidad ${ }^{10}$. En cambio los hemo- 


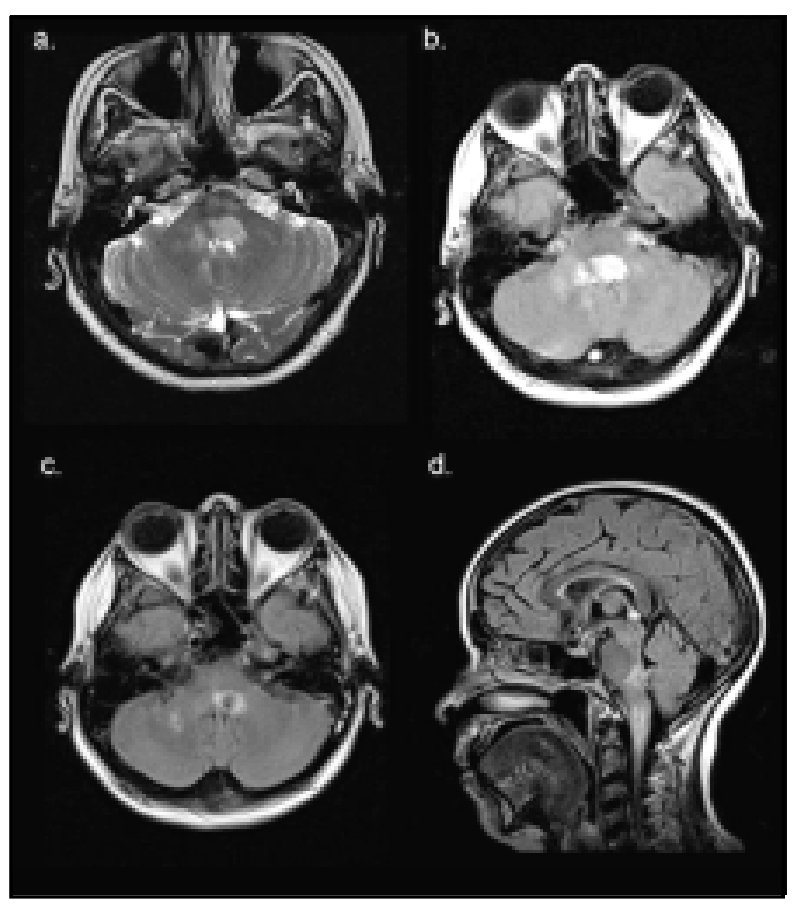

Figura 1. Las imágenes a (T2 Spin Echo) y b (T2 FLAIR) muestran una lesión hiperintensa con discreto efecto de masa en el aspecto posterior del tronco cerebral. En las imágenes c (T2 FLAIR) y d (T2 FLAIR sagital) se muestra otro paciente, con lesiones de forma alargada en el sentido céfalo caudal, una característica que se repitió en los distintos casos, y que presentaron realce homogéneo tras administración de gadolinio (no mostrado).

cultivos realizados con sistemas automatizados presentan una mayor sensibilidad ${ }^{11}$, hallazgos que fueron concordantes en nuestros casos clínicos en donde los cultivos de LCR fueron negativos y los hemocultivos positivos.

\section{Discusión}

La romboencefalitis secundaria a Listeria monocytogenes es una entidad rara con síntomas iniciales inespecíficos al igual que los estudios de LCR. Debido a lo anterior el diagnóstico suele ser tardío, lo que puede empeorar el pronóstico debido al compromiso del troncoencéfalo que puede llevar a una falla respiratoria ${ }^{10}$.

Debido al aumento de los casos de Listeria monocytogenes detectados en nuestro país ${ }^{12}$ hemos visto un número inusual de casos de romboencefalitis, experiencia compartida con otros centros.
Los casos presentados demuestran un perfil clínico similar con cuadro clínico prodrómico que en general dura alrededor de una semana a lo que se suman síntomas y signos de compromiso del tronco cerebral, hallazgos descritos en los reportes nacionales e internacionales ${ }^{10,13}$.

Los estudios de LCR son inespecíficos, describiéndose un aumento de los leucocitos pudiéndose encontrar indistintamente un predominio de polimorfonucleares o linfocitos. Las proteínas pueden o no estar elevadas. La glucosa suele ser normal. Estos hallazgos no siempre están presentes y cuando lo están pueden ser confundidos con una meningitis viral o bacteriana ${ }^{14}$. Se ha demostrado una mala sensibilidad de los cultivos de LCR para listeria así como también del Gram directo ${ }^{15}$.

Distinto es el caso de los hemocultivos, los que realizados adecuadamente suelen ser positivos y nos permiten certificar el diagnóstico. Es importante destacar la necesidad de informar al 


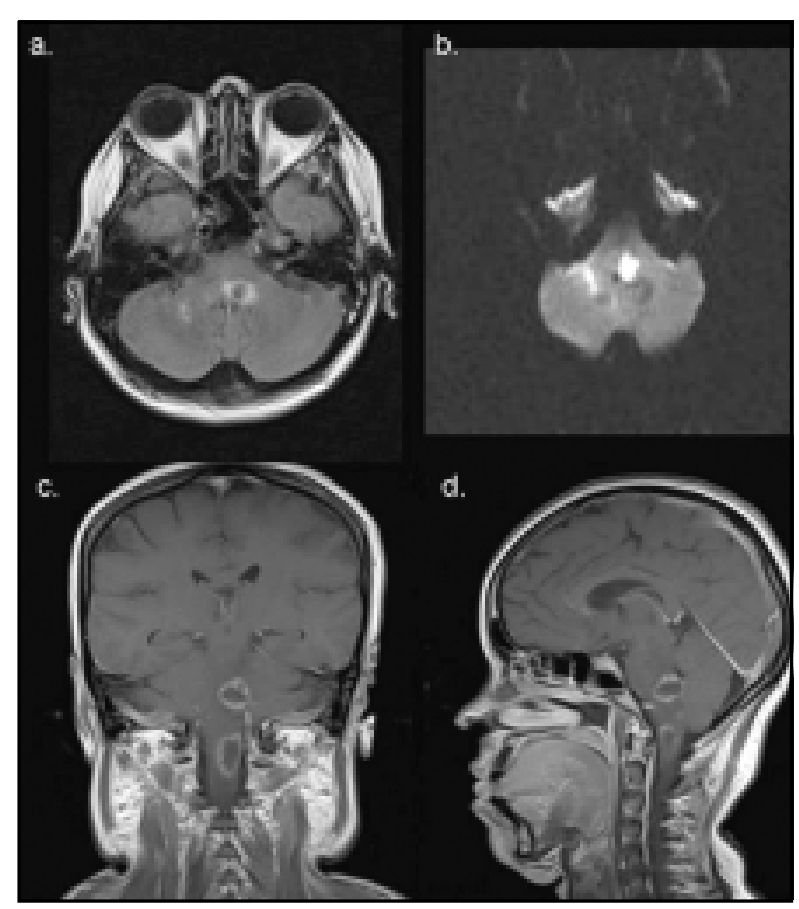

Figura 2. Evolución de las lesiones a formación de abscesos. Imagen a (T2 FLAIR) lesión hiperintensa con un centro isointenso, que muestra una clara restricción al movimiento de los protones en el estudio difusión apareciendo hiperintensa (figura b). También es posible identificar una pequeña lesión en el aspecto posterior del pedúnculo cerebeloso medio derecho. Las figuras c y d correspondientes a secuencias T1 con medio de contraste (gadolinio) donde se puede objetivar un claro realce anular en relación a los abscesos.

laboratorio la sospecha de Listeria monocytogenes, ya que requiere condiciones especiales, cultivos enriquecidos, no siempre realizados en forma rutinaria por los distintos laboratorios o idealmente realizar cultivos automatizados ${ }^{11,15}$.

Mención especial merecen los estudios imagenológicos por resonancia magnética los cuales son una herramienta esencial en el diagnóstico y seguimiento de estos pacientes. Como hemos mencionado previamente la romboencefalitis por Listeria monocytogenes produce característicamente lesiones unilaterales del tronco cerebral, comprometiendo a la protuberancia en relación al piso del cuarto ventrículo y con un diámetro mayor céfalo caudal. Estas lesiones presentan un realce heterogéneo con el medio de contraste. Esta es una característica que se repite en los distintos casos presentados en la literatura $7,9,16,17$. En los casos más avanzados se puede observar compromiso de pedúnculos cerebrales, cerebelo, bulbo e incluso médula espinal con o sin formación de abscesos cerebrales, los cuales como cualquier otro absceso producen una hiperintensidad en los estudios de difusión.

El diagnóstico diferencial imagenológico debe realizarse con otros agentes que producen romboencefalitis como el virus herpes simple y el adenovirus. Cabe mencionar también la tuberculosis y enfermedades mitóticas que pueden producir lesiones similares. Otro espectro de enfermedades que también pueden producir lesiones similares son las enfermedades desmielinizantes, cuyo tratamiento en base a corticoides puede conducir a un empeoramiento brusco de una romboencefalitis infecciosa. Estas patologías se pueden acompañar de otros hallazgos como el compromiso leptomenigeo de la tuberculosis o el compromiso de la sustancia blanca hemisférica y cuerpo calloso como puede ocurrir en las enfermedades desmielinizantes ${ }^{16-18}$. 
A pesar de lo amplio que puede parecer el diagnóstico diferencial pensamos que las imágenes de la romboencefalitis por Listeria monocytogenes tienen hallazgos que son bastante característicos y que se repiten en las distintas publicaciones, lo que sumado a un cuadro clínico y ausencia de otros hallazgos imagenológicos nos permiten realizar un diagnóstico bastante certero y así poder realizar un tratamiento empírico precoz, ya que los resultados de los estudios bacteriológicos pueden demorar un par de días y el tratamiento precoz puede disminuir la morbimortalidad de esta patología ${ }^{12}$.

\section{REFERENCIAS}

1. Kiara M, Posfay-Barbe, Eulen R. Listeriosis. Pediatr Rev 2004; 25: 151-9.

2. Stone S, Shoenberger J. Update on emerging infections: news from the centers for disease control and prevention. Ann Emer Med 2001; 38: 340-1.

3. MANDELL G. Principles and practice of infectious disease. V edition, 2000; LORVER B (cap 195) 2009-11.

4. Geun BG, Broome CV. Listeriorsis. JAMA 1989; 261: 1313-20.

5. López L, Gaztelurrutia L, Martínez I, Aguirrebengoa K, VALLS A, HeRNÁNDEZ JL ET aL. Listeriosis. Estudio de 16 años en un hospital terciario en España. Rev Panam Infectol 2007; 9: 30-7.

6. Rossi ML, Paiva A, Tornese M, Chianelu S, Troncoso A. Brotes de infección por Listeria monocytogenes. Una revisión de las vías que lleva a su aparición. Rev Chil Infect 2008; 25: 328-35.

7. Zagardo MT, Shanholtz CB, Zoarski GH, Rothman MI. Rhombencephalitis Caused by Adenovinus: MR Imaging Appearance. AJNR Am J Neuroradiol 1998; 19: 1901-3.

8. Chanqueo L, Gutiérrez C, Armas R, Urriola G, Bustos M, TAPIA C, VÁsQuez P. Bacteriemia primaria por Listeria monocytogenes en paciente con cirrosis hepática. Caso clínico. Rev Méd Chile 2008; 136: 225-9.

9. Kohler J, WinkLeR T, WAKhloo AK. Listeria brainstem encephalitis: two own cases and literature review. Infection 1991; 19: 36-40.

\section{CONCLUSIÓN}

La romboencefalitis por Listeria monocytogenes es una patología grave que requiere un diagnóstico y tratamiento precoz. Creemos que un adecuado grado de sospecha clínica y los estudios imagenológicos de $\mathrm{RM}$ son esenciales para el diagnóstico correcto, hallazgo que deben ser posteriormente confirmados con los estudios bacteriológicos. Las características imagenológicas en RM son bastante características y muchas veces son la clave para el diagnóstico correcto.

10. Illanes S, Araya P, Ferrer S, Cartier L, Bravo M, CASTILO S. Romboencefalitis: una forma de infección por Listeria monocytogenes en el sistema nervioso central. Rev Méd Chile 2003; 131: 921-8.

11. Wilson ML, Weinstein MP, ReLer LB. Automated blood culture systems. Clin Lab Med 1994; 14: 149-69.

12. Alper G, Knepper L, Kanal E. MR findings in listerial rhombencephalitias. AJNR 1996; 17: 593-6.

13. Noriega LM. Listeria monocytogenes: vieja bacteria, desafío permanente. Rev Chil Infect 2008; 25: 326-7.

14. Peeters A, Waer M, Michielsen P, Verbist l, Carton $H$. Listeria monocytogenes meningitis in adults: sixteen consecutive cases, 1973-1982. Scand J Infect Dis 1987; 19: 55-60.

15. Larraín D, Abarzúa F, De Jourdan F, Merino P, Belmar C, GarCía P. Infecciones por Listeria monocytogenes en mujeres embarazadas: experiencia del Hospital Clínico de la Pontificia Universidad Católica de Chile. Rev Chil Infect 2008; 25: 336-41.

16. Mrowka M, Graf L-P, Odin P. MRI findings in mesenrhombencephalitis due to Listeria monocytogenes. J Neurol Neurosurg Psychiatry 2002; 73: 775-80.

17. Soo MS, Tien RD, Gray L, Andrews PI, Friedman H. Mesenrhombencephalitis: MR findings in nine patients. AJNR 1993; 160: 1089-93

18. ARmstrong RW, Fung PC. Brainstem encephalitis (rhombencephalitis) due to Listeria monocytogenes: case report and review. Clin Infect Dis 1993; 16: 689-702. 